\title{
Correlation of Inflammatory Cells in Induced Sputum and Peripheral Blood of Patients with Acute Exacerbation of Chronic Obstructive Pulmonary Disease
}

\author{
Xin Wang1,2, Di Wu ${ }^{3}$, Yanmei Wu1,3, Hongyan Liu1, Ying Wang1,3, Chunmei Yun 1,3, Dejun Sun ${ }^{1,2,3}$, \\ Xiaoyu Gao ${ }^{*}$ \\ ${ }^{1}$ Key Laboratory of National Health Commission for the Diagnosis \& Treatment of COPD, Inner Mongolia Key Laboratory of \\ Respiratory Diseases, Inner Mongolia People's Hospital, Hohhot, China \\ ${ }^{2}$ Department of Geriatric Medicine, Inner Mongolia People's Hospital, Hohhot, China \\ ${ }^{3}$ Department of Respiratory and Critical Care Medicine, Inner Mongolia People’s Hospital, Hohhot, China \\ Email: *xiaoyugao2015@hotmail.com
}

How to cite this paper: Wang, X., Wu, D., Wu, Y.M., Liu, H.Y., Wang, Y., Yun, C.M., Sun, D.J. and Gao, X.Y. (2021) Correlation of Inflammatory Cells in Induced Sputum and Peripheral Blood of Patients with Acute Exacerbation of Chronic Obstructive Pulmonary Disease. Open Journal of Respiratory Diseases, 11, 105-115.

https://doi.org/10.4236/ojrd.2021.113010

Received: July 22, 2021

Accepted: August 24, 2021

Published: August 27, 2021

Copyright $\odot 2021$ by author(s) and Scientific Research Publishing Inc. This work is licensed under the Creative Commons Attribution International License (CC BY 4.0).

http://creativecommons.org/licenses/by/4.0/ (c) (i) Open Access

\begin{abstract}
Chronic obstructive pulmonary disease (COPD) is a major chronic respiratory disease worldwide. Inflammatory cells reflect the inflammatory situation both in peripheral blood and induced sputum. Their correlation has not been reported. The correlation between neutrophils (Neu), eosinophils (Eos), and lymphocyte (Lym) in induced sputum and that in peripheral blood of COPD patients was evaluated to explore the consistency of inflammatory cells in peripheral blood and induced sputum. Peripheral blood and induced sputum were collected from 437 patients with acute exacerbation of COPD (AECOPD) who were hospitalized in the Department of respiratory and critical care medicine, Inner Mongolia People's Hospital. The correlation analysis was performed by Spearman correlation analysis. The ratios of Neu, Eos, and Lym in induced sputum were $(79.15 \pm 22.60) \%,(5.23 \pm 12.74) \%$, and $(1.69 \pm 2.66) \%$. The ratios of Neu, Eos, and Lym in peripheral blood were $(63.29 \pm 11.44) \%,(2.99 \pm$ $3.60) \%$, and $(25.16 \pm 10.19) \%$. The results showed that the ratios of Neu and Eos in induced sputum were significantly correlated with the proportion of corresponding cells in peripheral blood $(\mathrm{P}<0.05)$. There was no correlation between the ratio of Lym and Leu in induced sputum and corresponding cells in peripheral blood $(P>0.05)$. In patients with AECOPD, the tendency of Neu and Eos in induced sputum was consistent with the corresponding cells in peripheral blood. Neu and Eos in induced sputum and peripheral blood reflected the degree of inflammation to guide the individualized medication of patients.
\end{abstract}




\section{Keywords}

Chronic Obstructive Pulmonary Disease, Induced Sputum, Peripheral Blood, Inflammatory Cells

\section{Introduction}

Chronic obstructive pulmonary disease (COPD) is a common preventable chronic airway disease caused by long-term exposure to risk factors [1], which is characterized by persistent respiratory symptoms and irreversible airflow limitation [2]. COPD has become a serious global public health burden [3], ranking third as a global cause of death by the World Health Organization in 2016 [4]. The pathogenesis of COPD is complex and diverse, among which inflammatory cell mechanism has been shown to play an important role [5] [6]. The harmful components in cigarette smoke enter the lung tissue, causing the activation of a series of inflammatory cells, such as neutrophil (Neu), eosinophils (Eos), Lymphocyte (Lym), leading to a chronic inflammatory response in the lung tissue [7] [8] [9]. The long-term inflammatory stimulation eventually leads to airway remodeling and irreversible airflow restriction [10]. Respiratory infection is an important cause of acute exacerbation of COPD (AECOPD) [11]. The airway inflammation and systemic inflammation response of patients in AECOPD are significantly enhanced than in stable-phase COPD patients, resulting in an increase in the number of inflammatory cells in peripheral blood. Eosinophils and neutrophils are increasingly being recognized as an important characteristic feature of COPD. COPD patients with eosinophilic inflammation respond to steroid therapy. However, eosinophilic inflammation may be defined based on various sampling techniques, including eosinophil levels in blood, sputum, bronchoalveolar lavage or biopsy, which leads to inconsistencies in its definition [12]. Blood eosinophils may increase in conjunction with sputum eosinophils during COPD exacerbations and therefore may be a good surrogate marker of airway eosinophilic inflammation. The count of Eos, Neu and Lym in peripheral blood can effectively respond to the changes of systemic inflammation in COPD patients. The induced sputum can effectively respond to the changes of airway inflammation in COPD by counting the cells in the airway and classifying the types of inflammation. It plays an important role in the evaluation of the disease condition and drug efficacy. Currently, there are many studies on the biomarkers in peripheral blood and induced sputum of COPD patients [13] [14], but there is no report on the relationship between peripheral blood cells and induced sputum cells. This study aimed to explore the consistency between the ratios of Neu, Eos, and Lym in induced sputum and those in peripheral blood of patients with COPD and to clarify the clinical significance of inflammatory cells in sputum and peripheral blood in COPD. 


\section{Materials and Methods}

\subsection{Research Objects}

The study was a cross-sectional study processed at the Department of Respiratory and Critical Care Medicine Inner of Mongolia People's Hospital from January 2015 to December 2015. 437 patients with AECOPD were recruited according to the guideline of Global Initiative for Chronic Obstructive Lung Disease (GOLD) 2013. All patients were diagnosed with COPD for more than one year. We excluded patients if they had a history of other respiratory illnesses, such as asthma, interstitial lung disease, lung cancer and pulmonary tuberculosis. Peripheral blood and induced sputum were collected from all patients on the first day of admission, and any oral or/and inhaled corticosteroids (ICS) was stopped 48 hours before the examination. Through structured questionnaire, the investigator conducted one-to-one inquiry. The questionnaire included age, gender, height, weight, Past diseases and smoking. After the examination all patients received routine treatment to prevent exacerbation in hospital, including antibiotics, bronchodilators, inhaled corticosteroids and other routine doses. At admission, some patients were unable to complete the pulmonary function test due to the exacerbation of COPD, so we did not grade the severity of the patients. The study was approved by the ethics committee of the people's Hospital of Inner Mongolia Autonomous Region, and all the subjects signed the informed consent.

\subsection{Sample Collection and Detection}

Peripheral blood collection and detection We collected 3 - $5 \mathrm{~mL}$ of venous blood using an Ethylene Diamine Tetraacetic Acid (EDTA) anticoagulant tube and were sent to the department of clinical laboratory for a routine blood test.

Induced sputum collection and detection The induced sputum samples were obtained after 20 - 30 minutes of atomization inhalation of $3 \%$ saline. The sputum without salivary components was put into a centrifuge tube and treated with $0.1 \%$ dithiothreitol. The cells were incubated at $37^{\circ} \mathrm{C}$, shaken, filtered, centrifuged, smeared with cell sediment, dried, stained with Swiss stain, counted at least 500 non-squamous cells: neutrophils, eosinophils, macrophages, lymphocytes, and epithelial cells. The sputum qualification criteria were as follows: sputum smears were regarded as qualified sputum samples, which were $<10$ squamous epithelial cells, $>25$ white blood cells/low power field, and weight of sputum without saliva component $>1 \mathrm{~g}$. If the cell survival rate was less than $50 \%$ and/or the squamous cell contamination was more than $20 \%$, the specimen was considered unqualified.

\subsection{Statistical Analyses}

Statistical analysis was made using software SPSS version 24.0 (IBM Corporation, Armonk, NY, USA). Data were expressed as mean \pm standard deviation (SD) and percent (\%) where appropriate. The correlation analysis was performed by Spearman correlation analysis. The difference between groups was compared 
by $t$-test. $\mathrm{p}<0.05$ was considered statistically significant.

\section{Results}

\subsection{Baseline}

In Table 1, A total of 437 patients with AECOPD were enrolled, comprising 285 males $(65.22 \%)$ and 152 females $(34.78 \%)$, with an average age of $69.30 \pm 9.02$ years. The ratios of Neu, Eos, and Lym in induced sputum were (79.15 \pm $22.60) \%,(5.23 \pm 12.74) \%$, and $(1.69 \pm 2.66) \%$. The ratios of Neu, Eos, and Lym in peripheral blood were $(63.29 \pm 11.44) \%,(2.99 \pm 3.60) \%$, and $(25.16 \pm$ $10.19) \%$. There were significant differences in Eos\%, Neu\%, and Lym\% between induced sputum and peripheral blood (Eos\%: $\mathrm{p}=0.00042$, Neu\%: $\mathrm{p}<0.0001$, and Lym\%: $\mathrm{p}<0.0001)$.

\subsection{Correlation Analysis of Neu, Eos, and Lym in Induced Sputum and Peripheral Blood}

The results showed that the ratios of Neu and Eos in induced sputum were significantly correlated with the proportion of corresponding cells in peripheral blood (Eos: $r=0.3925, p<0.0001$; Neu: $r=0.1579, p=0.0009$ ). There was no correlation between the ratio of Lym in induced sputum and corresponding cells in peripheral blood $(p=0.9295$, Figure 1$)$.

\subsection{Correlation Analysis of Peripheral Blood Leukocytes and Neu, Eos, Lym in Induced Sputum}

There was no correlation between the ratio of Leukocytes in peripheral blood and the ratio of inflammatory cells in induced sputum ( $p>0.05$, Figure 2$)$.

Table 1. Characteristics of AECOPD patients.

\begin{tabular}{ccc}
\hline Variable & COPD patient & p value \\
\hline Subjects, $\mathrm{n}$ & 437 & - \\
Male, $\mathrm{n}(\%)$ & $285(65.22 \%)$ & - \\
Age, yrs & $69.30 \pm 9.02$ & - \\
BMI & $22.16 \pm 4.08$ & - \\
Smoking index (pack-year) & $533.85 \pm 457.90$ & - \\
FEV1\% & $59.73 \pm 23.16$ & $<0.0001^{* * *}$ \\
FEV1/FVC & $57.11 \pm 11.95$ & \\
Neu\% in induced sputum & $79.15 \pm 22.60$ & $0.00042^{* * *}$ \\
Neu\% in peripheral blood & $63.29 \pm 11.44$ & \\
Eos\% in induced sputum & $5.23 \pm 12.74$ & $<0.0001^{*}$ \\
Eos\% in peripheral blood & $2.99 \pm 3.60$ & \\
Lym\% in induced sputum & $1.69 \pm 2.66$ & \\
Lym\% in peripheral blood & $25.16 \pm 10.19$ & \\
\hline
\end{tabular}

Data are shown as mean \pm SEM. Smoking index, number of cigarettes/days $\times$ years of smoking history. Neu\%: the ratios of neutrophils; Eos\%: the ratios of eosinophils; Lym\%: the ratios of lymphocytes. 


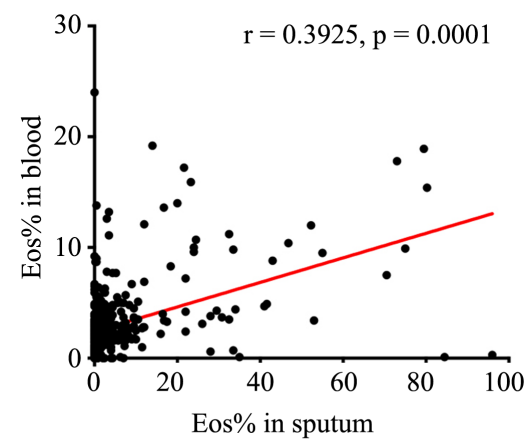

(a)

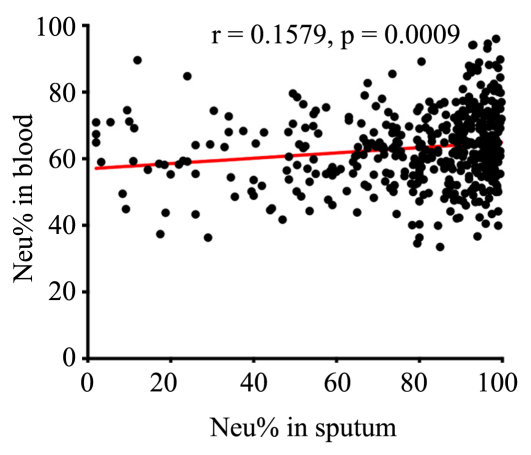

(b)

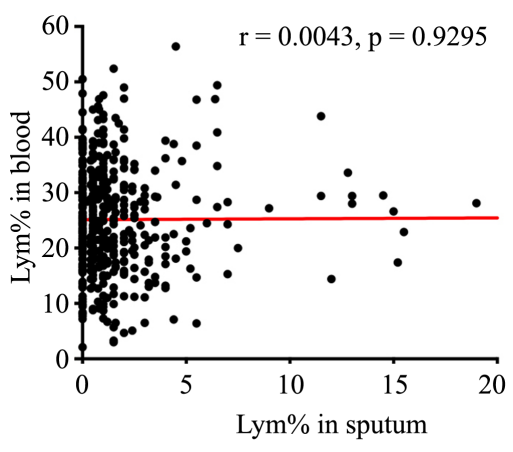

(c)

Figure 1. Correlation analysis of Eos\%, Neu\%, and Lym\% in induced sputum and peripheral blood. a. Neu\%: the ratios of neutrophils; Eos\%: the ratios of eosinophils; Lym\%: the ratios of lymphocytes.

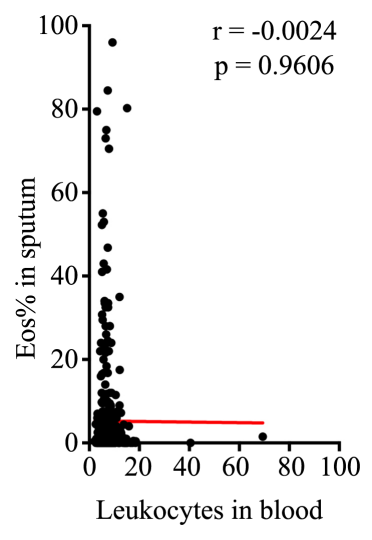

(a)

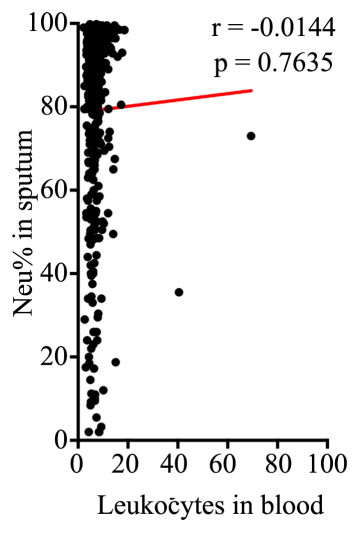

(b)

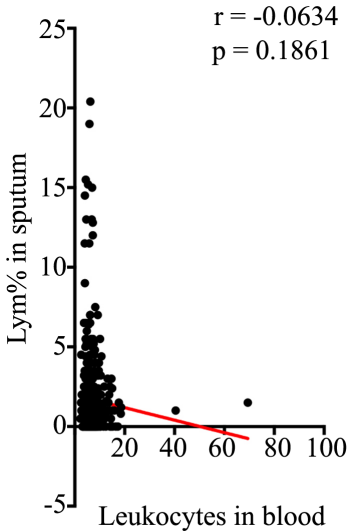

(c)

Figure 2. Correlation analysis of leukocytes in peripheral blood and Eos\%, Neu\%, Lym\% in induced sputum. Neu\%, the ratios of neutrophils, Eos\%, the ratios of eosinophils, Lym $\%$, the ratios of lymphocyte.

\section{Discussion}

COPD is a chronic airway inflammation characterized by incompletely reversible airflow limitation. For a long time, bronchoalveolar lavage and bronchial mucosa biopsy were the main methods to study airway inflammation. However, their application was seriously limited because of the low tolerance and compliance of patients. As a non-invasive and safe examination method, induced sputum cytology could effectively evaluate the severity of airway inflammation [15]. Compared with the blood samples from the whole body, the activated effective cells of induced sputum mediated by the related inflammation were higher, and the accuracy and repeatability were better. It also provided a simpler examination method for determine the treatment phenotype of patients with COPD to reduce the rate of misdiagnosis caused by atypical symptoms. According to the ratios of inflammation cells in induced sputum, airway inflammation could be divided into neutrophilic type, eosinophilic type mixed granulocyte type and pauci-granulocyte type. Then individual medication can be further guided so that COPD, a heterogeneous disease, could be better treated. This 
study evaluated the correlation between Neu, Eos, and Lym in induced sputum and that in peripheral blood of AECOPD patients and found that the ratios of $\mathrm{Neu}$, and Eos in induced sputum were consistent with the corresponding cells in peripheral blood, but there was no correction with the total number of blood leukocytes.

Neutrophils can mediate emphysema through protease/antiproteinase imbalance, regulate the continuous progress of chronic inflammation through delayed apoptosis, and release elastase and reactive oxygen species to mediate airway mucus hypersecretion and airway remodeling [16] [17], which are known to play a key role in the progress of COPD. The increased number of neutrophils in the airway of patients with acute exacerbation also indicates that neutrophils play an important role in the inflammatory response mechanism of COPD [18]. The results showed that induced sputum neutrophils were positively correlated with blood neutrophils, and the increased level of induced sputum neutrophils was significantly higher than that of peripheral blood neutrophils, indicating that induced sputum neutrophils could better reflect the degree of inflammation in patients with acute exacerbation than blood neutrophils, and were consistent with peripheral blood neutrophils. Jie Gao et al. found that a weaker correlation was between the percentage of sputum neutrophils and blood neutrophil percentage in AECOPD [19]. It was in accordance with our findings. They also reported that blood neutrophil ratio was decreased in mixed granulocytic AECOPD compared with neutrophilic AECOPD, which may reflect systemic inflammation. It was reported that the neutrophils ratio in induced sputum was significantly correlated with onset of COPD exacerbation [20]. The deterioration of COPD patients is usually related to neutrophilia and, to a lesser extent, eosinophilic airway inflammation. Previous studies have found that the ratio of neutrophils in sputum may be a biomarker to evaluate the status of COPD, that is, systemic inflammation, health status, COPD severity and future risk of exacerbation [21]. The neutrophil percentage in sputum was associated with the severity and life quality of COPD patients, but the neutrophil percentage was not associated with the exacerbation of COPD. This suggested that an induced sputum test was a good method to evaluate the airway inflammatory reaction and degree of acute exacerbation. In this study, the leukocyte count in peripheral blood to cells percentage in induced sputum was also analyzed, but no positive results were obtained. We presumed that that may be related to the influence of many factors on leukocytes.

The inflammation of AECOPD showed typically neutrophilic type, but eosinophils also existed and increased, its role in the inflammation of AECOPD is still uncertain [22]. This study found that the ratio of induced sputum eosinophils in COPD patients was significantly higher than that in peripheral blood and was positively correlated with blood eosinophils, which was consistent with the results of ProboszczM et al. [23] this suggested that induced sputum eosinophils may help identify sputum eosinophilia in AECOPD. The result is contrary 
to Jie Gao et al. [19] A study had also reported that blood eosinophils were a weak predictor of sputum eosinophils, and there was poor correlation between eosinophils in induced sputum and the corresponding cell in peripheral blood [24]. Thus, it is not certain whether sputum eosinophils can exactly predict peripheral eosinophilia in the AECOPD. Previous studies have suggested that eosinophils in blood and airway are significantly increased in patients with AECOPD [25]. Sputum eosinophilia is related to virus infection [26]. The presence of eosinophils indicates that patients with COPD could have a good response to bronchodilators and corticosteroids [27]. The assessment of eosinophils provides the better guidance to the use of corticksteroids especially in the prevention of some exacerbation. The GOLD 2021 [28] pointed out that blood eosinophil count can help clinicians to evaluate the possibility of beneficial preventive response after adding inhaled corticosteroids (ICS) to conventional bronchodilator treatment. Therefore, blood eosinophil count can be used as a biomarker in combination with clinical evaluation when making decisions on the use of ICS. The study found that sputum eosinophils in COPD patients was positively correlated with blood eosinophils, but it is uncertain sputum eosinophil count can be used as a biomarker to guide the use of ICS. It has been suggested that deterioration associated with sputum or blood eosinophilia may be more sensitive to systemic steroids [29]. The mechanisms for increased eosinophil count in patients with COPD are uncertain, but there has recently been considerable interest in the role of type 2 innate lymphoid cells (ILC2s), which might be important not only in intrinsic asthma but also in COPD [30] [31]. In addition, Studies found that compared with patients with asthma and COPD, patients with asthma COPD overlap had a higher percentage of eosinophils in sputum and lower level of lung function [23]. Inhaled corticosteroids could significantly improve the level of lung function and reduce the percentage of eosinophils in patients with asthma COPD overlap [27]. The presence of eosinophils in patients with COPD predicted a more favorable therapeutic response to bronchodilators and corticosteroids and indicated coexisting asthma or asthma-COPD overlap [27] [32].

The elevated number of $\mathrm{T}$ lymphocytes is found in lung parenchyma and airway of COPD patients, with a greater increase in CD8+ than CD4+ cells, and the number of $\mathrm{T}$ lymphocytes is known to be related to the amount of alveolar destruction and the severity of airflow obstruction [33]. Studies have confirmed that the main difference in inflammatory cell infiltration between asymptomatic smokers and COPD smokers is the increase in T lymphocytes, mainly CD8+ cells [34]. Long-term active or passive smoking causes $\mathrm{T}$ lymphocyte dysfunction, suppresses humoral and cellular immunity, and shows that the level of $\mathrm{T}$ lymphocyte subsets in smokers is low [35]. In addition, it has been pointed out that an imbalance both in T-lymphocyte subpopulation (CD4/CD8) and in CD8+ cell subsets $(\mathrm{Tc} 1 / \mathrm{Tc} 2)$ characterizes the inflammatory responses of smokers with COPD [36]. In this study, we only found that the level of lymphocytes in induced sputum was significantly lower than that in peripheral blood, and no 
correlation was found between induced sputum and lymphocytes in the blood. We speculated that the low level of lymphocytes in the sputum better reflected the immunosuppression of COPD than that in blood lymphocytes, and further information is required to evaluate the correlation between lymphocytes and COPD.

Thus, as a safe, effective, and reproducible examination method, induced sputum cytology can effectively evaluate the severity and type of COPD patients, detect the important inflammatory markers in the airway of patients, and then guide the individualized and accurate medication of COPD patients, and help to predict the prognosis of patients. It is of great significance to reduce the frequency of acute exacerbation of patients with COPD, improve the quality of life of patients and reduce the social and medical burden.

Our study investigated the relationship between inflammatory cells in sputum and peripheral blood and found that the ratios of Neu and Eos in induced sputum were significantly correlated with the proportion of corresponding cells in peripheral blood. Some patients were in the acute stage at admission and cannot complete the pulmonary function examination, thus we did not obtain complete pulmonary function data and could not analyze with the data of sputum and blood samples. We also did not conduct further investigation and follow-up on patients in stable stage, and we were unable to compare the data of patients in acute and stable stage. Another limitation is that we did not retain the biological samples collected, so we were unable to further study some inflammatory markers and inflammatory cells in the later stage.

\section{Data Availability Statement}

All data are available upon request to the corresponding authors.

\section{Funding}

This work was supported by the Non-profit Central Research Institute Fund of Chinese Academy of Medical Sciences (2019PT350001), National Natural Science Foundation of China (81960013), Natural Science Foundation of Inner Mongolian, China (2017BS0214), Joint Fund of Science and Technique Project supported by Millions of Inner Mongolian Medical University (YKD2016KJBW(LH)029), Foundation of Inner Mongolian People’s Hospital (2016079).

\section{Conflicts of Interest}

The authors declare that there are no conflicts of interest regarding the publication of this article.

\section{References}

[1] Pleasants, R.A., Heidari, K., Wheaton, A.G., Ohar, J.A., Strange, C., Croft, J.B., et al. (2015) Targeting Persons with or at High Risk for Chronic Obstructive Pulmonary Disease by State-Based Surveillance. COPD, 12, 680-689. 
[2] (2019) Global Strategy for the Diagnosis, Management and Prevention of COPD, Global Initiative for Chronic Obstructive Lung Disease (GOLD).

[3] Collaborators GBDCRD (2017) Global, Regional, and National Deaths, Prevalence, Disability-Adjusted Life Years, and Years Lived with Disability for Chronic Obstructive Pulmonary Disease and Asthma, 1990-2015: A Systematic Analysis for the Global Burden of Disease Study 2015. The Lancet Respiratory Medicine, 5, 691-706. https://doi.org/10.1016/S2213-2600(17)30293-X

[4] (2018) Global Health Estimates 2016: Deaths by Cause, Age, Sex, by Country and by Region, 2000-2016.

[5] Vogelmeier, C.F., Criner, G.J., Martinez, F.J., Anzueto, A., Barnes, P.J., Bourbeau, J., et al. (2017) Global Strategy for the Diagnosis, Management, and Prevention of Chronic Obstructive Lung Disease 2017 Report. GOLD Executive Summary. American Journal of Respiratory and Critical Care Medicine, 195, 557-582. https://doi.org/10.1164/rccm.201701-0218PP

[6] Barnes, P.J. (2016) Inflammatory Mechanisms in Patients with Chronic Obstructive Pulmonary Disease. The Journal of Allergy and Clinical Immunology, 138, 16-27. https://doi.org/10.1016/j.jaci.2016.05.011

[7] Strzelak, A., Ratajczak, A., Adamiec, A. and Feleszko, W. (2018) Tobacco Smoke Induces and Alters Immune Responses in the Lung Triggering Inflammation, Allergy, Asthma and Other Lung Diseases: A Mechanistic Review. International Journal of Environmental Research and Public Health, 15, 1033. https://doi.org/10.3390/ijerph15051033

[8] Hellermann, G.R., Nagy, S.B., Kong, X., Lockey, R.F. and Mohapatra, S.S. (2002) Mechanism of Cigarette Smoke Condensate-Induced Acute Inflammatory Response in Human Bronchial Epithelial Cells. Respiratory Research, 3, 22. https://doi.org/10.1186/rr172

[9] Churg, A., Zay, K., Shay, S., Xie, C., Shapiro, S.D., Hendricks, R., et al. (2002) Acute Cigarette Smoke-Induced Connective Tissue Breakdown Requires Both Neutrophils and Macrophage Metalloelastase in Mice. American Journal of Respiratory Cell and Molecular Biology, 27, 368-374. https://doi.org/10.1165/rcmb.4791

[10] Wang, Y., Xu, J., Meng, Y., Adcock, I.M. and Yao, X. (2018) Role of Inflammatory Cells in Airway Remodeling in COPD. International Journal of Chronic Obstructive Pulmonary Disease, 13, 3341-3348. https://doi.org/10.2147/COPD.S176122

[11] Ritchie, A.I. and Wedzicha, J.A. (2020) Definition, Causes, Pathogenesis, and Consequences of Chronic Obstructive Pulmonary Disease Exacerbations. Clinics in Chest Medicine, 41, 421-438. https://doi.org/10.1016/j.ccm.2020.06.007

[12] Oliver, B., Tonga, K., Darley, D., Rutting, S., Zhang, X., Chen, H., et al. (2019) COPD Treatment Choices Based on Blood Eosinophils: Are We There Yet? Breathe (Sheffield, England), 15, 318-323. https://doi.org/10.1183/20734735.0254-2019

[13] Tangedal, S., Nielsen, R., Aanerud, M., Persson, L.J., Wiker, H.G., Bakke, P.S., et al. (2019) Sputum Microbiota and Inflammation at Stable State and during Exacerbations in a Cohort of Chronic Obstructive Pulmonary Disease (COPD) Patients. PLoS ONE, 14, e0222449. https://doi.org/10.1371/journal.pone.0222449

[14] Aaron, S.D., Vandemheen, K.L., Ramsay, T., Zhang, C., Avnur, Z., Nikolcheva, T., et al. (2010) Multi Analyte Profiling and Variability of Inflammatory Markers in Blood and Induced Sputum in Patients with Stable COPD. Respiratory Research, 11, 41. https://doi.org/10.1186/1465-9921-11-41

[15] Bakakos, P., Schleich, F., Alchanatis, M. and Louis, R. (2011) Induced Sputum in Asthma: From Bench to Bedside. Current Medicinal Chemistry, 18, 1415-1422. 
https://doi.org/10.2174/092986711795328337

[16] Sinden, N.J. and Stockley, R.A. (2013) Proteinase 3 Activity in Sputum from Subjects with Alpha-1-Antitrypsin Deficiency and COPD. European Respiratory Journal, 41, 1042-1050. https://doi.org/10.1183/09031936.00089712

[17] Jasper, A.E., McIver, W.J., Sapey, E. and Walton, G.M. (2019) Understanding the Role of Neutrophils in Chronic Inflammatory Airway Disease. F1000Research, 8, F1000 Faculty Rev-557. https://doi.org/10.12688/f1000research.18411.1

[18] Paliogiannis, P., Fois, A.G., Sotgia, S., Mangoni, A.A., Zinellu, E., Pirina, P., et al. (2018) Neutrophil to Lymphocyte Ratio and Clinical Outcomes in COPD: Recent Evidence and Future Perspectives. The European Respiratory Review, 27, Article ID: 170113. https://doi.org/10.1183/16000617.0113-2017

[19] Gao, J., Chen, B., Wu, S. and Wu, F. (2020) Blood Cell for the Differentiation of Airway Inflammatory Phenotypes in COPD Exacerbations. BMC Pulmonary Medicine, 20, 50. https://doi.org/10.1186/s12890-020-1086-1

[20] Kinose, D., Ogawa, E., Kudo, M., Marumo, S., Kiyokawa, H., Hoshino, Y., et al. (2016) Association of COPD Exacerbation Frequency with Gene Expression of Pattern Recognition Receptors in Inflammatory Cells in Induced Sputum. The Clinical Respiratory Journal, 10, 11-21. https://doi.org/10.1111/cri.12171

[21] Hurst, J.R., Vestbo, J., Anzueto, A., Locantore, N., Mullerova, H., Tal-Singer, R., et al. (2010) Susceptibility to Exacerbation in Chronic Obstructive Pulmonary Disease. The New England Journal of Medicine, 363, 1128-1138. https://doi.org/10.1056/NEJMoa0909883

[22] Mycroft, K., Krenke, R. and Gorska, K. (2020) Eosinophils in COPD-Current Concepts and Clinical Implications. The Journal of Allergy and Clinical Immunology: In Practice, 8, 2565-2574. https://doi.org/10.1016/j.jaip.2020.03.017

[23] Proboszcz, M., Mycroft, K., Paplinska-Goryca, M., Gorska, K., Nejman-Gryz, P., Jankowski, P., et al. (2019) Relationship between Blood and Induced Sputum Eosinophils, Bronchial Hyperresponsiveness and Reversibility of Airway Obstruction in Mild-to-Moderate Chronic Obstructive Pulmonary Disease. COPD, 16, 354-361. https://doi.org/10.1080/15412555.2019.1675150

[24] Hastie, A.T., Martinez, F.J., Curtis, J.L., Doerschuk, C.M., Hansel, N.N., Christenson, S., et al. (2017) Association of Sputum and Blood Eosinophil Concentrations with Clinical Measures of COPD Severity: An Analysis of the SPIROMICS Cohort. The Lancet Respiratory Medicine, 5, 956-967.

https://doi.org/10.1016/S2213-2600(17)30432-0

[25] Bafadhel, M., McKenna, S., Terry, S., Mistry, V., Reid, C., Haldar, P., et al. (2011) Acute Exacerbations of Chronic Obstructive Pulmonary Disease: Identification of Biologic Clusters and Their Biomarkers. American Journal of Respiratory and Critical Care Medicine, 184, 662-671. https://doi.org/10.1164/rccm.201104-05970C

[26] Papi, A., Bellettato, C.M., Braccioni, F., Romagnoli, M., Casolari, P., Caramori, G., et al. (2006) Infections and Airway Inflammation in Chronic Obstructive Pulmonary Disease Severe Exacerbations. American Journal of Respiratory and Critical Care Medicine, 173, 1114-1121. https://doi.org/10.1164/rccm.200506-859OC

[27] Postma, D.S. and Rabe, K.F. (2015) The Asthma-COPD Overlap Syndrome. The New England Journal of Medicine, 373, 1241-1249. https://doi.org/10.1056/NEJMra1411863

[28] (2021) Global Strategy for the Diagnosis, Management and Prevention of COPD, Global Initiative for Chronic Obstructive Lung Disease (GOLD).

[29] Bafadhel, M., McKenna, S., Terry, S., Mistry, V., Pancholi, M., Venge, P., et al. 
(2012) Blood Eosinophils to Direct Corticosteroid Treatment of Exacerbations of Chronic Obstructive Pulmonary Disease: A Randomized Placebo-Controlled Trial. American Journal of Respiratory and Critical Care Medicine, 186, 48-55. https://doi.org/10.1164/rccm.201108-1553OC

[30] Scanlon, S.T. and McKenzie, A.N. (2012) Type 2 Innate Lymphoid Cells: New Players in Asthma and Allergy. Current Opinion in Immunology, 24, 707-712.

https://doi.org/10.1016/j.coi.2012.08.009

[31] De Grove, K.C., Provoost, S., Verhamme, F.M., Bracke, K.R., Joos, G.F., Maes, T., et al. (2016) Characterization and Quantification of Innate Lymphoid Cell Subsets in Human Lung. PLOS ONE, 11, e0145961.

https://doi.org/10.1371/journal.pone.0145961

[32] Bateman, E.D., Reddel, H.K., van Zyl-Smit, R.N. and Agusti, A. (2015) The Asthma-COPD Overlap Syndrome: Towards a Revised Taxonomy of Chronic Airways Diseases? The Lancet Respiratory Medicine, 3, 719-728. https://doi.org/10.1016/S2213-2600(15)00254-4

[33] Hogg, J.C., Chu, F., Utokaparch, S., Woods, R., Elliott, W.M., Buzatu, L., et al. (2004) The Nature of Small-Airway Obstruction in Chronic Obstructive Pulmonary Disease. The New England Journal of Medicine, 350, 2645-2653.

https://doi.org/10.1056/NEJMoa032158

[34] Costa, C., Rufino, R., Traves, S.L., Lapa, E.S.J.R., Barnes, P.J. and Donnelly, L.E. (2008) CXCR3 and CCR5 Chemokines in Induced Sputum from Patients with COPD. Chest, 133, 26-33. https://doi.org/10.1378/chest.07-0393

[35] Barnes, P.J. (2017) Cellular and Molecular Mechanisms of Asthma and COPD. Clinical Science (London, England: 1979), 131, 1541-1558. https://doi.org/10.1042/CS20160487

[36] Tzanakis, N., Chrysofakis, G., Tsoumakidou, M., Kyriakou, D., Tsiligianni, J., Bouros, D., et al. (2004) Induced Sputum CD8+ T-Lymphocyte Subpopulations in Chronic Obstructive Pulmonary Disease. Respiratory Medicine, 98, 57-65. https://doi.org/10.1016/j.rmed.2003.08.007 